\title{
Sleep Onset Problems in Two Children with Mild Intellectual Disability and Epilepsy: Assessment and Treatment in the Home Setting
}

\author{
A.P.H.M. Maas ${ }^{1,3}$, R. Didden ${ }^{1}$, J.M.H. de Moor ${ }^{1,2}$, W.O. Renier ${ }^{2}$ \& L.M.G. Curfs ${ }^{3}$ \\ Radboud University Nijmegen, \\ Radboud University Nijmegen Medical Centre $e^{2}$, \\ Maastricht University Hospital ${ }^{3}$, \\ The Netherlands
}

\begin{abstract}
Sleep problems such as bedtime difficulties, frequent night waking and excessive daytime sleepiness are prevalent in children with epilepsy. In the present study, functional assessment of sleep onset problems in two young children with epilepsy was performed. Effects of bedtime fading and antipsychotic medication (pipamperon) in a 6-year-old boy, and melatonin in an 8-year-old girl were assessed on sleep latency. Treatment resulted in a marked decrease in sleep latency in both cases and effects were maintained after three months. Keywords: Sleep problems, epilepsy, developmental disabilities, bedtime fading, melatonin, anti-psychotic medication.
\end{abstract}

\section{Introduction}

Children with developmental disabilities are at increased risk for developing sleep problems. Several studies have shown that sleep problems are much more prevalent in children with developmental disabilities than in their nondisabled peers. An important risk factor for developing sleep problems is the presence of a seizure disorder. An association between sleep problems and epilepsy has been found in correlational (see e.g., Didden, Korzilius, Van Aperlo, Van Overloop, \& De Vries, 2002; Quine, 1991) and quasi-experimental studies (see e.g., Stores, Wiggs, \& Campling, 1998). Stores et al. compared sleep behaviors of 79 children with mild forms of epilepsy with healthy children from the general population matched for age and gender. They found that, compared to their controls, children with epilepsy showed significant higher rates of sleep disorder symptoms, especially those suggesting poor sleep quality and sleep-related anxieties. More recently, in a sample of 66 children with different types of epilepsy De Moor, Didden, Korzilius \& Renier (in preparation) found that: (a) children with epilepsy have more sleep problems than those in a control group, (b) type of epilepsy was related to sleep problems (e.g. children with maligne epilepsy showed more sleep fragmentation than those with other types of epilepsy), and (c) sleep problems were positively related to severity of epileptic attacks. Furthermore, they found that both the severity of epilepsy and daytime problem behaviors were positively associated with sle ep problems.

Results of the above studies show that children with epilepsy are at increased risk for developing sleep problems. In most, if not all cases, both the presence of sleep problems and epilepsy have detrimental effects on the well-being of the child and his or her parent(s). Children may show daytime fatigue, irritability and hyperactivity and noncompliance. Furthermore, sleep problems, and especially sleep deprivation is associated with decreased cognitive functioning and learning during academic tasks. Many parents report fatigue and symptoms of depression as a result of longstanding sleep problems in their child.

Given the adverse consequences of sleep problems, assessment and treatment are warranted. Undoubtedly, scientific knowledge about the treatment of sleep problems in children with developmental disabilities has increased during the past decades. Chronic sleep problems in children with developmental disabilities are usually complex, in that such problems may be caused by psychological and/or medical factors. Treatment of sleep problems includes various behavioral methods, such as stimulus control and sleep hygiene, extinction, sleep restriction, bedtime fading and chronotherapy. Medically oriented treatment include treatment of physical cause of sleep problems 
(e.g., reflux, pain), surgery (e.g., removal tonsils and adenoids in case they cause airway obstruction) and pharmacological approaches (e.g., melatonin, sedative medication) (for a review, see Didden \& Sigafoos, 2001). It is recommended that a functional assessment of the sleep problems be conducted to identify possible relationships between disruptive sleep behaviors and parental management techniques (Didden, Curfs, Van Driel, \& De Moor, 2002). Functional assessment may provide clues for behavioral treatment. If no such relationships are identified, other types of treatment are indicated.

In the present study, behavioral and pharmacological treatments were implemented to address severe and chronic sleep onset problems in two young children who had a history of seizures and who were mildly intellectually disabled. In each case, functional assessment of the sleep onset problems preceded treatment. Whereas a link between sleep behaviors and parental management was identified in one child, such a link could not be identified in the other child. Treatment consisted of bedtime fading combined with antipsychotic medication for one child (Ken) and melatonin for the other child (Ellis).

\section{Method}

Participants and setting

Ken was 6 years and 5 months old and had a history of partial seizures. He was free of seizures as a result of which anti-epileptic medication was terminated 10 months ago. He still had mild absences during the day. He was diagnosed as mildly intellectually disabled and visited a special education classroom for children with autism spectrum disorders. He was the only child of a singleparent family. During the day Ken was very active.

Ellis was a 8 years and 3 months old girl with complex partial epilepsy and mild intellectual disability. She received $250 \mathrm{mg}$ levetiracetam (Keppra ${ }^{\circledR}$ ) in the morning and evening and $100 \mathrm{mg}$ lamotrigine (Lamictal®) in the morning and $75 \mathrm{mg}$ in the evening (5 p.m.). She had absences on a daily basis, mainly in the mornings. She attended a special education classroom for children with learning difficulties and was the only child in a two-parent family. During the day Ellis was very active, especially in the evening.

Both children were referred to the medical centre of the Radboud University Nijmegen for treatment of sleep problems. Functional assessment and treatment of sleep problems were conducted in the child's home setting.

Description of sleep problems

At bedtime (6.15 p.m.), Ken refused to go to bed and was very busy. After his mother eventually succeeded in putting Ken into bed, he frequently got out of it. Contingent on getting out of bed, his mother told him to go back to bed to sleep. As a result, he usually fell asleep around 11 p.m. Ken woke up three or more times a night. Every night he had to go to the toilet and a few times a week he woke up in panic and was screaming and sweating. According to his mother Ken was restless during his sleep and talked a lot while asleep. He also showed teeth grinding and snoring. Sometimes his mother had to wake him at 6.30 a.m. on schooldays. During the day he showed symptoms of tiredness, usually starting at 3 p.m., but mostly stayed awake. Ken showed sleep problems since he was born. When he was 3.5 years old he was prescribed melatonin (dose unknown) for treatment of his sleep problems. According to his mother the sleep problems became less intense. Although getting off to sleep remained problematic, he didn't wake up at night once asleep as a result of this treatment. After two years, mother decided to terminate melatonin treatment because of its limited effectiveness.

Ellis went to bed willingly, but showed problems getting off to sleep almost every evening. When her father took her to bed at 8 p.m., she was wide awake. Usually she stayed in bed, but it took 1 to 2 hours for her to fall asleep. According to her mother Ellis was restless during her sleep. However, she usually didn't wake at night and in the morning parents had to wake her at 7 a.m. 
Although she was still tired in the morning, she didn't take naps during the day. Her sleep problems became more apparent when Ellis was 7.5 years old.

Both children showed severe sleep onset problems that existed for at least 9 months prior to baseline recordings. Epilepsy and/or antiepileptic medication were ruled out as cause for maintenance of children's sleep problems. Dosage and timing of the anti-epileptic medication remained constant throughout the study.

Functional assessment

Prior to baseline, the participant's mother filled out a questionnaire and an interview was conducted with each mother. Information was obtained on the child's development and medical history, emergence of sleep problems, type and duration of sleep problems and situations in which sleep onset problems and night waking were (not) likely to occur. In addition, each participant's mother completed a standardized sleep log daily during two weeks of baseline. If disruptive bedtime behavior occurred during the evening and/or night, she was instructed to record its antecedent and consequent events.

Treatment procedures

Prior to treatment, parents were informed about appropriate sleep routines (stimulus control, sleep hygiene). The results of functional assessment and the rationale of treatment were explained to them.

A functional relationship between Ken's disruptive sleep behaviors and mother's behaviors was identified during functional assessment. Despite mother's attempts to send Ken back to bed immediately, Ken succeeded every night in postponing his bedtime by getting out of bed. Besides, he didn't seem sleepy at bedtime. Consequently, there was no association between being put into bed and falling asleep. Therefore a behavioral treatment seemed appropriate. Ken's highly disruptive bedtime behavior and mother being a single parent considered, bedtime fading was more suitable for treatment than an extinction procedure. Ken's mother was instructed to discontinue her usual management techniques. The principles of bedtime fading were explained to her. The rationale of this behavioral treatment was to keep him awake until he falls asleep on his own. Bedtime was delayed to the time at which he was willing to go to bed. When the new bedtime was successful (i.e., no disruptive behavior at bedtime) the bedtime was faded back stepwise, that is in steps of 15 minutes. Bedtime fading started at 10.30 p.m. and remained in effect until a bedtime of 8.00 p.m. was established. Considering the fact that Ken was highly active in the evening and his sleep problems had lasted for many years, bedtime fading was supported by $4 \mathrm{mg}$ of pipamperon given to him 1 hour before being put into bed. Pipamperon is an antipsychotic with fatigue and sleepiness as side-effects.

Ellis was willing to go to bed at bedtime and did not get out of bed or disturb her parents. Because there were no problems in her bedtime routine (that is, no adverse parental reinforcement was identified during functional assessment) and Ellis was wide awake at bedtime, a disturbance in melatonin secretion was hypothesized. The pineal hormone melatonin plays a key role in the synchronization of circadian rhythms, especially the sleep-wake and temperature rhythms. Melatonin secretion starts in the evening, peaks at about 2:00 a.m. and reaches its basic levels again early in the morning. A delay in melatonin secretion corresponds with a delayed sleep-wake rhythm. Exogenous melatonin administered a couple of hours before the onset of endogenous melatonin secretion advances the sleep-wake rhythm (Smits et al., 2003). Therefore, treatment for Ellis consisted of oral melatonin $(3 \mathrm{mg})$ given to her about 1,5 hours before being put into bed.

At the start of treatment parents were daily given feedback by the principal therapist (first author) over the phone. Later on the frequency of telephone calls was faded to once every week or once every two weeks. 
Recording

A standardized sleep log was used daily during all experimental conditions. On this sheet, the participant's mother recorded time put into bed, sleep onset time (evening), night waking, sleep offset time (morning) and antecedents and consequences of disturbing behaviors, if any. The main dependent variable was sleep latency (in minutes). Sleep latency was calculated by subtracting sleep onset time by the time at which the child was put into bed. Sleep onset problems were defined as severe if sleep latency was more than 60 minutes (Wiggs \& Stores, 1996).

Apart from the sleep log, other pre- and post-treatment measures were used to assess participants' daytime behaviors and parenting situation regarding the participants' sleep. Daytime behaviors were evaluated by means of the Child Behavior Checklist - Dutch Version (CBCL; Verhulst, Koot, Akkerhuis \& Veerman, 1990). Normalized T-scores for Total problems scores, Internalizing score and Externalizing score were computed. The $95^{\text {th }}$ percentile $(T$-score $>60)$ is used as the cutoff point of significant clinically deviant behavior.

Part B of the Nijmegen Questionnaire on Child-rearing Situations (NQCS; Wels \& Robbroeckx, 1996) was administered to investigate judgments of parents regarding their own parenting situation. Parents are instructed to indicate which of eight descriptions fits best their own situation. In the first situation, no problems in child-rearing are present and support is absolutely unnecessary. In situation eight, child-rearing is highly problematic and parents are in urgent need of support. Small adjustments in the questionnaire were made, because we were particularly interested in the parents' judgment of the parenting situation regarding their child's sleep.

Design

For each child, data were collected in a single case design with follow-up. An AB-design was used for Ken and a reversal design was used for Ellis. For each child, follow-up data were collected 3 months after treatment. The participant's mother was asked to fill out a questionnaire about her satisfaction with the treatment for sleep onset problems (social validity) at follow-up. Furthermore, the participant's mother was asked to fill out the CBCL and NQCS (part B) during baseline, at the end of treatment and during follow-up.

Results

Figure 1 presents sleep latency of Ken during conditions of baseline, treatment and follow-up. The horizontal line marks the 60 minutes cutoff point for sleep onset problems. Mean sleep latency during baseline, bedtime fading plus $4 \mathrm{mg}$ pipamperon, bedtime fading plus $8 \mathrm{mg}$ pipamperon, last treatment phase (time put to bed 8 p.m.; from night 92 onwards) plus $8 \mathrm{mg}$ pipamperon, and followup with Ken was $134(S D=117.2), 56(S D=36.7), 33(S D=22.0), 14(S D=5.4)$, and $9(S D=3.5)$, respectively.

During baseline, Ken's sleep onset problems were present in $60 \%$ of the nights, in $36 \%$ of the nights during treatment plus $4 \mathrm{mg}$ pipamperon, in $6 \%$ during treatment plus $8 \mathrm{mg}$ pipamperon and in $0 \%$ during the last phase of treatment, and follow-up. At follow-up, Ken's mother was highly satisfied with treatment outcome. She considered this treatment to be appropriate and acceptable. 


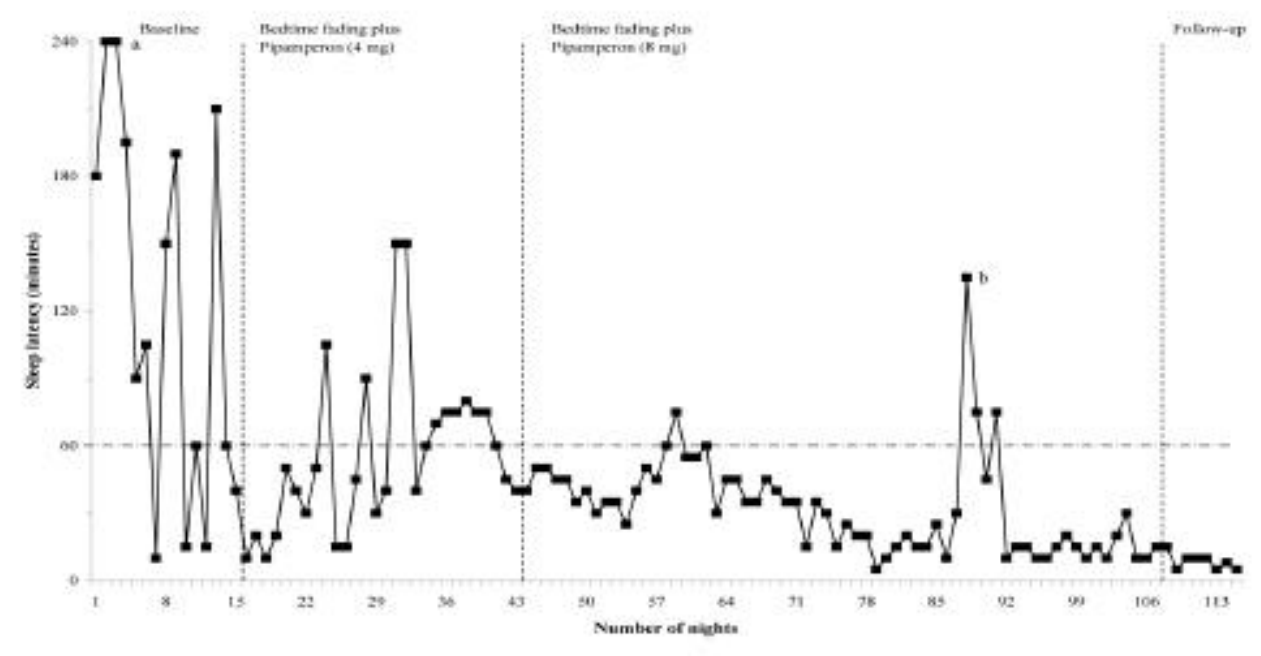

Figure 1: Sleep latency for Ken during conditions of baseline, treatment and follow-up ( $a=$ sleep latency: 450 minutes; $b=$ seizures)

Figure 2 shows that Ellis' mean sleep latency during the first baseline condition was 121 minutes $(S D=64.2)$. Melatonin resulted in a decrease to $26(S D=22.9)$. During a return to baseline, mean sleep latency increased to $94(S D=67.5)$. This mean showed a marked decrease after reinstatement of melatonin, with a mean of $32(S D=10.6)$. During follow-up, there was a further and lasting decrease in sleep latency to a mean of $21(S D=11.2)$. The dose of melatonin was reduced by half during follow-up.

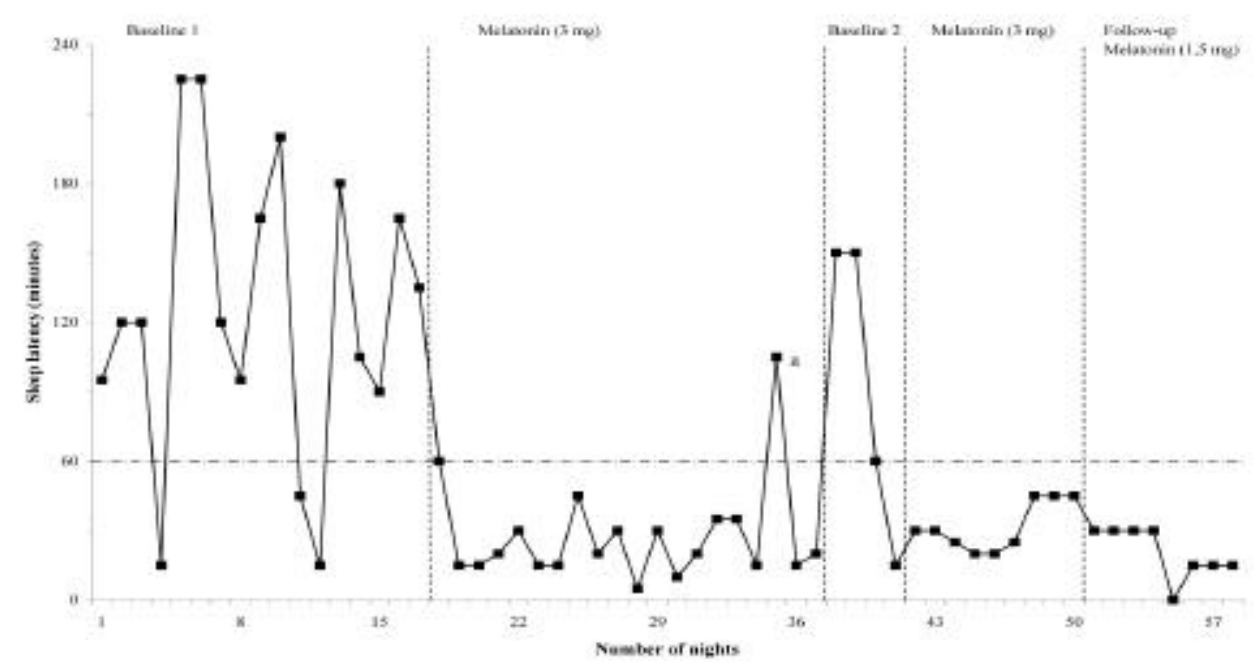

Figure 2: Sleep latency for Ellis during conditions of baseline, treatment, return to baseline, treatment and follow-up $(a=$ ill $)$ 
Table 1: Daytime behavior (CBCL, T-scores) and parenting situation regarding the child's sleep (NQCS, 1 = problem free, $8=$ very problematic) for Ken and Ellis during conditions of baseline, end of treatment and follow-up.

\begin{tabular}{|c|c|c|c|}
\hline & Baseline & End of treatment & Follow-up \\
\hline \multicolumn{4}{|l|}{ Ken } \\
\hline \multicolumn{4}{|l|}{ Child Behavior Checklist } \\
\hline Total problem score & $76^{*}$ & $71 *$ & $74 *$ \\
\hline Internalizing score & $65^{*}$ & $61 * *$ & $67 *$ \\
\hline Externalizing score & $71 *$ & $64 *$ & $66^{*}$ \\
\hline \multicolumn{4}{|l|}{$\begin{array}{l}\text { Nijmegen Questionnaire on Child- } \\
\text { rearing Situations, part B }\end{array}$} \\
\hline Opinion on parenting situation & 6 & 2 & 2 \\
\hline \multicolumn{4}{|l|}{ Ellis } \\
\hline \multicolumn{4}{|l|}{ Child Behavior Checklist } \\
\hline Total problem score & $67 *$ & 58 & 56 \\
\hline Internalizing score & $63 * *$ & 59 & 47 \\
\hline Externalizing score & 52 & 52 & 52 \\
\hline \multicolumn{4}{|l|}{$\begin{array}{l}\text { Nijmegen Questionnaire on Child- } \\
\text { rearing Situations, part B }\end{array}$} \\
\hline Opinion on parenting situation & 6 & 2 & 1 \\
\hline
\end{tabular}

** above the $95^{\text {th }}$ percentile, $*$ above the $98^{\text {th }}$ percentile

Her sleep onset problems were present during baseline 1, melatonin, baseline 2, melatonin and follow-up conditions in 78\%, 5\%,50\%, 0\%, and $0 \%$ of the nights, respectively. Also, Ellis' mother was highly satisfied with treatment outcome and considered this treatment very acceptable and appropriate.

As depicted in Table 1, Ken showed significant daytime behavior problems during baseline, at the end of treatment and follow-up (CBCL Total problem scores were above the $98^{\text {th }}$ percentile). Both internalizing and externalizing behavior problems were present in all conditions. On item level, 'Sleeps little', 'Talks, cries in sleep' and 'Sleep problems' were often applicable during baseline. At the end of treatment 'Nightmares' were sometimes applicable and 'Sleeps little' and 'Talks, cries in sleep' were still often applicable. 'Nightmares', 'Overtired', 'Sleeps little' en 'Sleep problems' were sometimes applicable at follow up and 'Talks, cries in sleep' was often applicable. In mother's opinion the parenting situation on Ken's sleep was problematic during baseline. However, at the end of treatment and at follow up she indicated that this situation was almost problem-free and she didn't need support.

Ellis showed significant daytime behavior problems during baseline (CBCL Total problem Tscore was above the $98^{\text {th }}$ percentile) and internalizing problems were present. Ellis' mother reported Attention problems, Somatic complaints ( $\mathrm{T}$-scores were above the $98^{\text {th }}$ percentile) and Social problems (T-score were above the $95^{\text {th }}$ percentile) more frequently than parents from the reference group. However, at the end of treatment and at follow-up there were no significant daytime behavior problems (CBCL Total problem T-score below the $95^{\text {th }}$ percentile). Attention problems and Social problems ( $\mathrm{T}$-scores above the $95^{\text {th }}$ percentile) were reported more frequently at the end of treatment and Attention problems ( $\mathrm{T}$-score above the $98^{\text {th }}$ percentile) at follow-up than for the reference group. On item level 'Overtired', 'Sleeps little' and 'Sleep problems' were often applicable during baseline. 'Overtired' was sometimes applicable at the end of treatment. At follow-up, no problem behaviors regarding sleep were reported. In her mother's opinion, the parenting situation on Ellis' sleep was problematic during baseline, but almost problem-free at the end of treatment; at follow-up, there were no problems at all and support was absolutely unnecessary.

Discussion 
In the present case studies, severe sleep onset problems were successfully treated using behavioral and/or pharmacological approaches. For each child, large sleep latencies (with mean sleep latencies during baseline larger than 120 minutes) were substantially reduced and effects of treatment were maintained. At follow-up the participant's mothers were satisfied about both outcome and type of treatment.

Bedtime disruptive behaviors of Ken may be partly explained by a dysfunctional bedtime routine. In Ken's case, one may hypothesize that because of chronic and severe sleep onset problems he had failed to learn to associate being put into bed with falling asleep. Another factor may be held responsible for his sleep onset problems, that is his activity level. During the first treatment phase (bedtime fading plus $4 \mathrm{mg}$ pipamperon) Ken frequently got out of bed and refused to return to bed. Pipamperon was used to increase fatigue and herewith reduce probability that Ken got out of his bed. When the dose of pipamperon was increased to $8 \mathrm{mg}$ he got out of bed only once a night between 11.00 p.m. and 1.00 a.m. to go to the toilet and went back to bed on his own. At follow-up, his mother reported that Ken slept well if bedtime was set around 8.00 p.m. and using $8 \mathrm{mg}$ of pipamperon. Giving him a lower dosage resulted in a worsening of his sleep onset problems. However, Ken's mother rated CBCL items on sleep problems ('Nightmares', 'Overtired', 'Sleeps little', 'Sleep problems' and 'Talks, cries in sleep') as 'sometimes' or 'often' (sleep talking) present at follow-up. His mother indicated that sleep problems were only present in highly exceptional situations, such as for example, when Ken expected a pleasant event to be happening the day after. Parasomnias, like sleep talking, night terrors and nightmares are usually transient and resolve without further specific treatment (see e.g., Didden \& Sigafoos, 2001).

Our hypothesis that a disturbance in melatonin secretion may have caused sleep onset problems in Ellis seems highly probable. The positive effect of oral melatonin suggests a disturbance in the circadian sleep-wake rhythm (delayed sleep-wake rhythm). A shortcoming is that we failed to investigate evening melatonin levels before and during treatment to empirically support this.

Other shortcomings should be mentioned. Internal validity of results in Ken cannot be claimed. The reversal design used for Ellis has more internal validity. In this case, it should be mentioned that sleep latency showed a decline at the end of the second baseline condition (no oral melatonin was given). Nevertheless, this baseline supports the positive effect of oral melatonin on the sleep-wake rhythm, because bedtime was belated in the 3rd and 4th night (night 40 and 41) of reversal until 10.00 and 11.00 p.m. respectively (in the 1 st and 2 nd night she fell asleep at 11.30 p.m.). Her parents wanted to continue the melatonin treatment, because epileptic absences in the morning became more frequent. As a result of this, Ellis got incontinent for urine and feces. At follow-up parents halved the dose to $1,5 \mathrm{mg}$ melatonin without consulting the principal therapist. Lowering the dose of melatonin, however, did not result in an increase of sleep latency in Ellis.

Treatment of sleep onset problems in two children with mild intellectual disability and epilepsy resulted not only in a substantial shorter sleep latency, but also in a significant improvement in daytime behavior with Ellis and in remediation of parenting stress in both families (as shown by results from rating scales, see Table 1). Anecdotal reports at follow-up from Ken's mother indicate that at daytime Ken was less irritable, more cheerful and he seemed to concentrate better at school. Ellis was reported to be more rested and refreshed upon waking in the morning. She still had daytime absences.

Treatment of sleep disorders in children with epilepsy should not only be directed at treatment of the epilepsy. In these children sleep problems are not necessarily a symptom of epilepsy. A multidisciplinary (psychological and medical) approach in treatment of sleep onset problems in children with mild intellectual disability and epilepsy is recommended. 


\section{References}

Didden, R., Curfs, L.M.G., Driel, S. van, Moor, J.M.H. de (2002). Sleep problems in children and young adults with developmental disabilities: home-based functional assessment and treatment. Journal of Behavior Therapy and Experimental Psychiatry, 33, 49-58.

Didden, R., Korzilius, H., Aperlo, B. van, Overloop, C. van, \& Vries, M. de (2002). Sleep problems and daytime problem behaviors in children with intellectual disability. Journal of Intellectual Disability Research, 46, 537-547.

Didden, R., \& Sigafoos, J. (2001). A review of the nature and treatment of sleep problems in individuals with developmental disabilities. Research in Developmental Disabilities, 22, $252-$ 274.

Moor, J.M.H. de, Didden, R., Korzilius, H., \& Renier, W.O. (in preparation). Epilepsy, sleep problems and daytime problem behavior in children who have a seizure disorder.

Quine, L. (1991). Sleep problems in children with mental handicap. Journal of Mental Deficiency Research, 35, 269-290.

Smits, M.G., Stel, H.F. van, Heijden, K. van der, Meijer, A.M., Coenen, A.M.L., \& Kerkhof, G.A. (2003). Melatonin improves health status in children with idiopatic chronic sleep-onset insomnia: A randomized placebo-controlled trial. Journal of the American Academy of Child and Adolescent Psychiatry, 42, 1286-1293.

Stores, G., Wiggs, L., \& Campling, G. (1998). Sleep disorders and their relationship to psychological disturbance in children with epilepsy. Child: Health, Care and Development, 24, 5-19.

Verhulst, F.C., Koot, J.M., Akkerhuis, G.W., \& Veerman, J.W. (1990). Praktische handleiding voor de CBCL. Assen: Van Gorcum.

Wels, P.M.A., \& Robbroeckx, L.M.H. (1996). Nijmeegse Vragenlijst voor de Opvoedingsbelasting (NVOS). Handleiding. Lisse: Swets \& Zeitlinger.

Wiggs, L., \& Stores, G. (1996). Severe sleep disturbance and daytime challenging behaviour in children with severe learning difficulties. Journal of Intellectual Disability Research, 40, 518528 .

Author Contact Information:

A.P.H.M. Maas, M.A.

Radboud University Nijmegen

Department of Special Education

P.O. Box 9104

6500 HE Nijmegen

The Netherlands

a.maas@pwo.ru.nl

Dr. R. Didden

Radboud University Nijmegen

Department of Special Education

P.O. Box 9104

6500 HE Nijmegen

The Netherlands

r.didden@pwo.ru.nl 
Prof. dr. J.M.H. de Moor

Radboud University Nijmegen

Department of Special Education

P.O. Box 9104

6500 HE Nijmegen

The Netherlands

j.demoor@pwo.ru.nl

Prof. dr. W.O. Renier

Radboud University Nijmegen Medical Centre

Department of Neurology

P.O. Box 9101

6500 HB Nijmegen

The Netherlands

Prof. dr. L.M.G. Curfs

Maastricht University Hospital

Department of Clinical Genetics

P.O. Box 5800

6202 AX Maastricht

The Netherlands

curfs@msm.nl

\section{ADVERTISING IN THE INTERNATIONAL JOURNAL OF BEHAVIORAL AND CONSULTATION THERAPY}

The prices for advertising in one issue are as follows:

1/4 Page: $\$ 50.00$ 1/2 Page: $\$ 100.00$ vertical or horizontal Full Page: $\$ 200.00$

If you wish to run the same ad in multiple issues for the year, you are eligible for the following discount:

1/4 Pg.: \$40 - per issue

1/2 Pg.: \$75 - per issue -vertical or horizontal

Full Page: \$150.00-per issue

An additional one time layout/composition fee of $\$ 25.00$ is applicable

For more information, or place an ad, contact Halina Dziewolska by phone at (215) 462-6737 or e-mail at: halinadz@ hotmail.com 